THE ASTROPHYSICAL JOURNAL SUPPLEMENT SERIES, 90:735-742, 1994 February

(c) 1994. The American Astronomical Society. All rights reserved. Printed in U.S.A.

\title{
ROSAT OBSERVATIONS OF STELLAR FLARES
}

\author{
J. H. M. M. SCHMITT \\ Max-Planck-Institut für Extraterrestrische Physik, D-85740 Garching bei München, Germany \\ Received 1993 February 24; accepted 1993 June 21
}

\begin{abstract}
X-ray observations of stellar flares obtained during the ROSAT all-sky survey as well as in the ROSAT pointing program are discussed. The ROSAT all-sky survey allowed-for the first time-an unbiased search for stellar flares among all types of stars. A fundamentally new result obtained is that flares can occur on all types of late-type stars, thus supporting the view that the $\mathrm{X}$-ray emission from these stars is controlled by magnetic processes. Long-duration flares can be studied with the all-sky survey data particularly well, and an especially well-observed long-duration flare event on the flare star EV Lacertae is presented and discussed in detail. Finally, the issue of time variability on the shortest detectable timescales and the question of microflaring is discussed using $R O S A T$ data from a pointed observation of UV Ceti.
\end{abstract}

Subject headings: stars: coronae — stars: flare — stars: late-type - X-rays: stars

\section{INTRODUCTION}

On the Sun the occurrence of flares, i.e., sudden eruptions of electromagnetic radiation, has been known for more than a hundred years; the realization, however, that flares constitute a primarily coronal rather than chromospheric or photospheric phenomenon, was a result of spaceborne observations mostly at $\mathrm{X}$-ray wavelengths. In the stellar context, the first flares were discovered in the late 1940's, and since then the number of known flare stars has been steadily increasing.

In the seventies, the first X-ray observations of stellar flares were made. A major breakthrough for stellar X-ray astronomy in general and X-ray studies of stellar flares in particular occurred with the availability of imaging $X$-ray telescopes such as the Einstein Observatory (operating between 1978-1981) and the EXOSAT Observatory (operating between 1983-1985). Both of these satellites were run as observatories and were used to monitor a few of the known (optical) flare stars for X-ray flares. Comprehensive reviews of the Einstein observations of stellar X-ray flares were presented by Haisch (1983) and of the EXOSAT observations by Pallavicini, Stella, \& Tagliaferri (1990a); a review of the current problems of solar and stellar flare research has been given by Haisch, Strong, \& Rodonò (1991). In addition, a smaller number of particularly well observed flares on individual objects have been published, for example, an Einstein Observatory observation of a flare on Proxima Centauri by Haisch et al. (1983), EXOSAT observations of flares on UV Ceti by deJager et al. (1986), on Algol and $\sigma$ CrB by van den Oord et al. (1988, 1989), and GINGA observations of flares on II Peg (Doyle et al. 1991), UX Ari (Tsuru et al. 1989), and Algol (Stern et al. 1991).

It is important to realize that (probably) all hitherto obtained X-ray observations of stellar flares relate to the detection of hot thermal plasma produced during the flare eruption. There are no observations of stellar flares in the hard X-ray or $\gamma$-ray range; the highest $X$-ray energies with detected $X$-ray emission are $\sim 15 \mathrm{keV}$ ( see Doyle et al. 1991), but it is believed that this radiation is still coming from hot thermal (rather than nonthermal) plasma. The evidence for the thermal character of the observed emission is, however, often quite indirect; the most direct evidence comes from the presence of the $6.7 \mathrm{keV}$ iron "line" observed in the GINGA spectra of flares on UX Ari (cf. Tsuru et al. 1989) and the EXOSAT ME spectra of EQ Peg (Poletto, Pallavicini, \& Kopp 1989). As a consequence, we have-in the stellar context-no direct evidence for particle acceleration as indicated by nonthermal X-ray emission. The question whether the hot thermal plasma, which we can observe and diagnose at soft X-ray energies, is relevant in the context of particle acceleration depends on the unknown heating process(es). If the heating occurs directly through particles, one would have at least a proxy indicator for acceleration processes in stellar environments; if not, there may be no link between soft X-ray emission and particle acceleration processes. On the Sun, there is in fact no one-to-one relationship between the soft X-ray properties and the hard X-ray and $\gamma$ ray properties of a flare; in general, however, flares with large soft X-ray energy output also tend to produce substantial nonthermal emission at higher energies, and flares exceeding the $G O E S$ classification $\mathrm{X} 10$, always show hard $\mathrm{X}$-ray and $\gamma$-ray emission (Rieger 1993).

In this article I will try to address various items relevant to studies of stellar flares with the ROSAT Observatory. First, I will demonstrate the contribution for the study of stellar flares specifically made by $R O S A T$ in the form of the all-sky survey; second, I will demonstrate by example my thesis that all types of late-type stars can entertain flares in their X-ray emission; third, I will give a detailed discussion of a long-duration flare event detected in the ROSAT all-sky survey on the nearby flare star EV Lacertae and the implications of these measurements for the physical properties of the flare plasma, and finally I will discuss new ROSAT results on the shortest timescales of detectable X-ray variability using pointed $R O S A T$ observations of the archetypical flare star UV Ceti.

\section{THE ROSAT CONTRIBUTION}

The ROSAT position sensitive proportional counter (PSPC) with its large collecting area at soft $\mathrm{X}$-ray energies is 
particularly well suited for the study of stellar flares. In addition, the ROSAT wide field camera (WFC) allowed for the first time the coverage of the EUV band, and indeed Barstow et al. (1992) report the detection of an EUV flare on the active binary system BY Dra. The ROSAT satellite is also operated in an observatory mode just like the Einstein and EXOSAT observatories, and a number of dedicated studies of flare stars have been undertaken. Some results of such studies on the nearby flare star UV Ceti will be reported below; however, since $R O S A T$ is still operating, it is far too early for a comprehensive assessment of pointed ROSAT observations of flare stars.

In addition to such pointed observations of selected flare stars, ROSAT carried out its all-sky survey in the time between 1990 July 30 and 1991 January 25. This all-sky survey was carried out in such a way that the sky was scanned along great circles with the ROSAT X-ray telescope; the scanning period was equal to the orbital period, i.e., $\sim 96$ minutes. The (ecliptic) longitude of the great circle of the instantaneous scan path moved with the apparent speed of the Sun along the plane of the ecliptic, i.e., $\sim 1$ degree per day. Since the PSPC field of view is 2 degrees, a given source was scanned for a minimum of 2 days, and correspondingly longer if located at higher ecliptic latitude. During a single scan, a given source remained in the field of view for typically $15-30$ seconds. From the point of view of an individual X-ray source, the all-sky survey can therefore be considered to consist out of a sequence of snapshots (with $\sim 15-30$ s integrations each) more or less equally spaced over an interval of at least 2 days.

Obviously, the all-sky survey observing mode is particularly well suited to study long-duration flares which are difficult to study otherwise with a satellite in low-earth orbit. Even more importantly, the all-sky survey really did cover the whole sky in an unbiased fashion. Therefore all types of stars can be checked for the occurrence of flares regardless of whether they are known to be flare stars or not, while the pointing program of $\operatorname{ROSAT}$ is heavily biased toward studying well-known flare stars.

\section{WHICH STARS FLARE IN X-RAYS?}

Magnetic fields, present in the outer envelopes of late-type stars, are thought to lie at the root of stellar activity as evidenced, for example, through $\mathrm{X}$-ray emission. These fields are required, first, to confine the hot $\mathrm{X}$-ray emitting coronal gas, and, second, they are also believed to be relevant for the coronal heating process( $\mathrm{es}$ ) heating the coronal plasma to X-ray emitting temperatures. In this context observations of X-ray flaring are then interpreted as an indicator that the observed activity is indeed related to magnetic fields (see Linsky 1985), since the conversion of (nonpotential) magnetic field energy into heat appears to be the only (reasonable) way by which the observed energy release can be accounted for.

One of the basic results of our studies of X-ray time variability is the fact that flaring X-ray emission can be found on all types of late-type stars; I emphasize that I do not claim that flares have been observed on all late-type stars during the allsky survey but rather examples of flares on all types of late-type stars can be produced. Attempts to detect time variability in early-type stars have so far been largely unsuccessful, adding credence to the usual assumption that the X-ray production mechanism in early-type stars is quite different from that operating in late-type stars ( see the discussion by Linsky 1985 on this issue).

The proof of my thesis occurs by way of example, and in the following I present some X-ray light curves containing flares, or refer the reader to light curves published elsewhere. The latest (in terms of spectral type) star so far found to exhibit flaring is the dM6e star AZ Cnc; the all-sky survey light curve of AZ Cnc is published by Fleming et al. (1993) in their Figure $2 a$. The light curve of $\mathrm{AZ} \mathrm{Cnc}$ is remarkable since $\mathrm{AZ}$ Cnc could be detected only because a long duration flare-persisting for about 10 hours-occurred at the beginning of the allsky survey observations. The recorded peak X-ray luminosity of this flare is $\sim 2.2 \times 10^{29} \mathrm{ergs} \mathrm{s}^{-1}$, which corresponds to $7.5 \%$ of the bolometric (quiescent) luminosity of the star; unfortunately there are no concurrent measurements of this flare at other wavelengths, but it is likely that the total flare luminosity constituted a significant fraction of the star's bolometric luminosity.

Many flares have been recorded on nearby dMe and $\mathrm{dKe}$ stars. The record holder in terms of peak X-ray flux is the 9.3 mag K0 V star HD 197890 (see Bromage et al. 1992, who nick-named this star "Speedy Mic"). During the all-sky survey a long-duration event with a peak flux of 65 PSPC counts $\mathrm{s}^{-1}$ (corresponding to an energy flux of $\sim 4 \times 10^{-10} \mathrm{ergs} \mathrm{s}^{-1} \mathrm{~cm}^{-2}$ ) was observed. Assuming a bolometric correction of 0.2 , one calculates a ratio between bolometric (quiescent) and X-ray luminosity of $8.2 \%$, which is even larger than the ratio of the flare on AZ Cnc. A detailed analysis of the "Speedy Mic" flare is performed by Kürster \& Schmitt (1994); the basic point to note here is that the rotation period of this star is shorter than the observed flare decay time scale and yet no eclipses by rotational modulation are observed. This indicates an organisation of the flare plasma on large spatial scales unless the flare occurred in the circumpolar region.

I have not been able to find any literature reports on X-ray flares from F-type stars. In Figure 1, I show the all-sky survey light curve of the F5 star 36 Dra. This light curve is unusual since it covers $\sim 40$ days of continuous X-ray observations; because of its high ecliptic latitude, 36 Dra was scanned for a

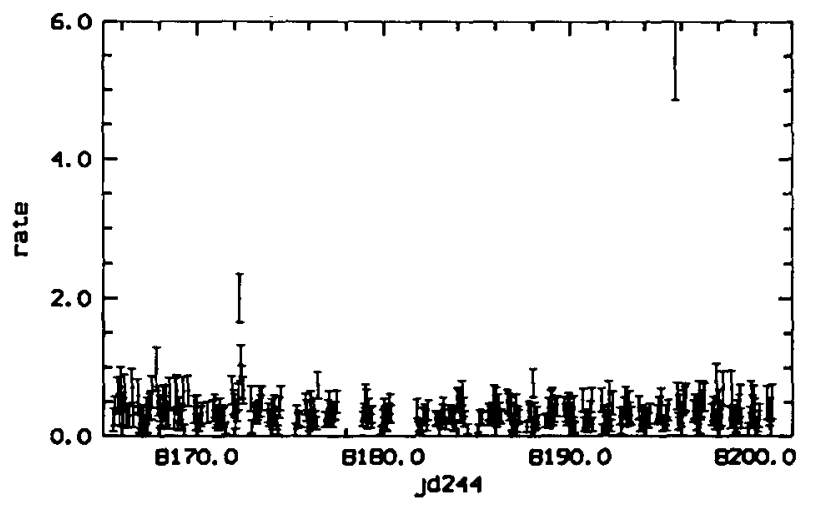

FIG. 1.-ROSAT all-sky survey light curve of the single F5 V star 36 Dra; two presumably short duration flares were detected during 40 days of scanning. 
good fraction of the ROSAT all-sky survey. 36 Dra has been detected in all of approximately 700 individual scans; two scans clearly stick out and indicate the occurrence of shortduration flare events. The larger one of the two flares corresponds to an instantaneous X-ray luminosity of $\sim 1.5 \times 10^{30}$ ergs $\mathrm{s}^{-1}$, using a distance of $21 \mathrm{pc}$ toward 36 Dra. Since only one scan was found high, we cannot estimate the decay time scale and hence the total radiative energy output; since the decay timescale must be less than 5600 s (i.e., ROSAT"s orbital period) and is unlikely to have been $100 \mathrm{~s}$ or less, an estimate of $\sim 1000 \mathrm{~s}$ should be correct to within an order of magnitude, implying a total radiative energy release in considerable excess of $10^{33}$ ergs.

A number of flares have been detected on stars of spectral type A and B; such stars are thought not to have outer convective envelopes and therefore not able to support magnetic field generation by electromagnetic dynamos. Nevertheless, some of these "early-type" stars do show coronal X-ray emission (see Schmitt et al. 1993b), and thus the appearance of X-ray flaring may not come totally unexpectedly. In particular, the occurrence of X-ray flares on $\alpha \mathrm{Gem}$ ( = Castor A/B; see Pallavicini et al. 1990b) was confirmed; the earliest star with detected X-ray flaring appears to be the B star (visual) binary pair $\pi$ Lup (=HR 5605/5606). In Figure 2, I show the all-sky survey light curve of $\pi$ Lup. The source is weak, but half a day before the end of the survey observations of $\pi$ Lup a huge flare occurred with a peak flux of $\sim 3.2 \times 10^{31} \mathrm{ergs} \mathrm{cm}^{-2} \mathrm{~s}^{-1}$. The total energy content of this event is difficult to estimate because of the rather sparse data coverage; however, the decay timescale is of the order of $\sim 5000 \mathrm{~s}$ so that the total radiative energy output $E_{\mathrm{rad}}$ of this event must be in excess of $10^{35} \mathrm{ergs}$. This large value of $E_{\mathrm{rad}}$ argues against the hypothesis that a hitherto unseen late-type companion may be responsible for the quiescent and flaring $\mathrm{X}$-ray emission, rather it seems as if $\mathrm{X}$-ray emission is coming from one or both B-type stars (see Schmitt et al. 1993b).

\section{A LONG-DURATION FLARE ON THE NEARBY FLARE STAR EV LACERTAE}

As an example of the potential of the ROSAT all-sky survey for the study of long-duration flares I will present an analysis of

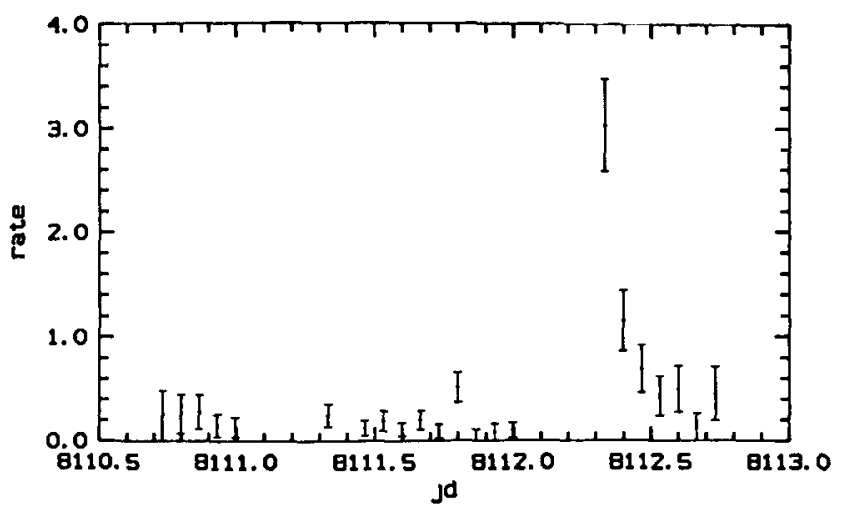

FIG. 2.- ROSAT all-sky survey light curve of the B star binary system HR 5605/HR 5606 ( $=\pi$ Lup). Note the large flare near the end of the all-sky survey observations; see text for details.

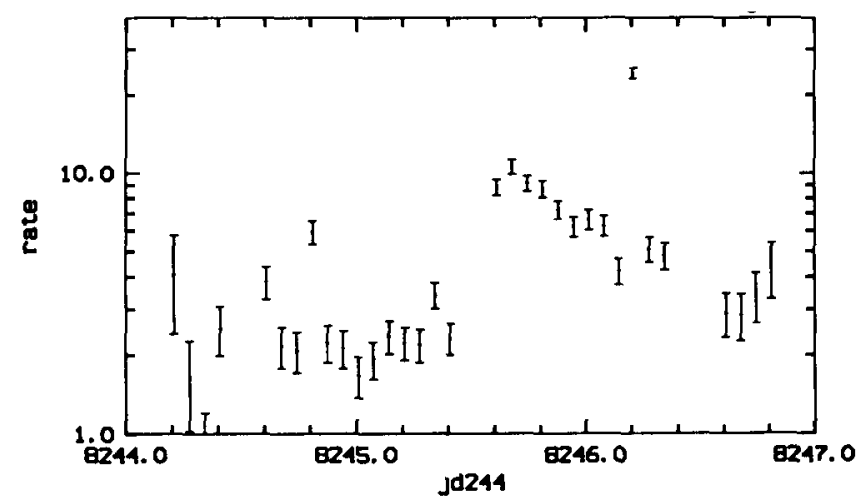

FIG. 3.- ROSAT all-sky survey light curve of the dMe dwarf EV Lacertae. Note the variety of states the star was found in during the all-sky survey observations: The first half of the observation was "quiescent", while the second half is characterized by a long-duration event, on top of which a long-short duration event occurred.

the all-sky survey data obtained for the nearby flare star EV Lac. This object is rather close to the Sun $(d=5 \mathrm{pc})$, and is known to be a strong emitter of both quiescent ( see Schmitt \& Rosso 1988) and flaring X-ray emission (Ambruster, Snyder, \& Woods 1984).

In Figure 3 (upper panel), I present the observed all-sky survey light curve for EV Lac. As can be seen from Figure 3, during the first half of the 3 day survey coverage EV Lac was more or less constant with a few occasional small flares occurring. The second half of the light curve is dominated by an enormous long-duration flare. Unfortunately one cannot locate the flare onset and hence the duration of the flare rise very accurately because there is a data gap between the last quiescent scan and the first flare scan. However, since the peak count rate is observed in the second flare scan, the rise time must have been at least 96 minutes (i.e., ROSAT's orbital period), while an upper limit of 290 minutes can be derived from the time elapsed between flare peak and the last quiescent scan. After the flare peak at JD 2448245.68, the count rate decreases roughly exponentially for the next 24 hours, with the exception of a single rather large short duration flare at JD 2448246.2, which exceeds the peak count rate of the long duration event by more than a factor of 3 . I will take the view that this short duration flare is unrelated to the long-duration event to be discussed here and ignore this data point in all subsequent analyses; as an aside I note that a GOES satellite at $1 \mathrm{AU}$ distance from EV Lac would have recorded this event as a $\mathrm{X} 140$ flare!

In Figure 4, I plot the measured PSPC hardness ratio (defined as the ratio between the count rate in the $0.5-2.4 \mathrm{keV}$ passband to the rate in the $0.1-0.3 \mathrm{keV}$ passband) for the EV Lac all-sky survey data. As can be seen from Figure 4, the limited statistics of the all-sky survey data ( $\sim 20$ s integrations $)$ leads to uncomfortably large errors especially for the quiescent emission. At any rate, no large changes in the spectral hardness are observed, with a possibility that during the flare (i.e., data points after JD 2448245.6) the spectral hardness is a little greater than during quiescence. An analysis of the PSPC pulse height spectrum accumulated during the quiescent period requires a two-temperature component description (with 


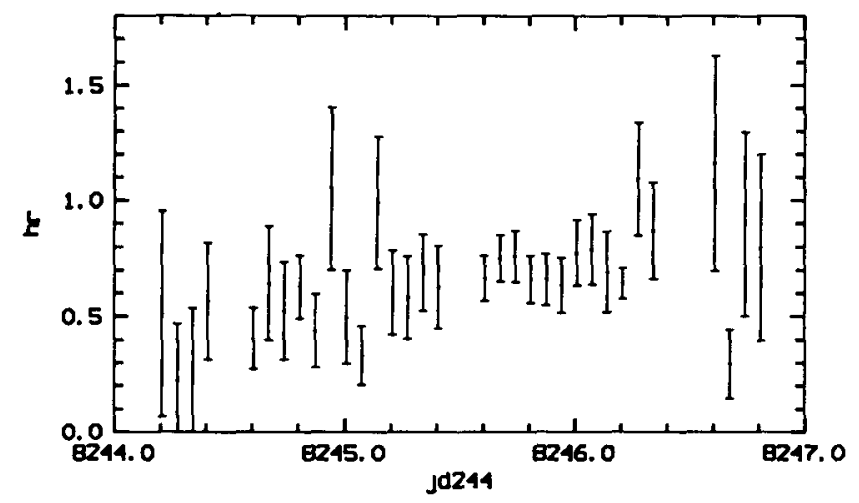

FIG. 4.-PSPC hardness ratio for the EV Lac long-duration flare; note that the hardness changes only little (if at all) while the intensity changes by an order of magnitude.

$\log T_{\text {high }}=6.99$ and $\log T_{\text {low }}=6.25$ in accordance with spectral fits of flare stars obtained with the Einstein Observatory IPC; see Schmitt et al. 1990). A detailed analysis of the PSPC pulse height spectra during the flare decay is severely hampered by the low signal-to-noise ratio of these spectra. The spectra are, however, consistent with rather high temperatures, with a best fit yielding a temperature of $\log T_{\text {flare }}=7.4$. Such high temperatures are consistent with flare temperatures determined from EXOSAT and GINGA measurements; however, for good temperature determinations one really needs pointing data with much better count statistics.

\subsection{Modeling the Long-duration Flare on EV Lac}

In the following section I will present the results of simple modeling attempts of the light curve of the long duration flare on EV Lac. The basic goal of these investigations is to interpret the flare data in the context of physically consistent models and infer basic parameters about the flaring plasma. As we will see shortly, such modeling does not lead to unique models, and quite different physical pictures about the flare emerge depending on the assumed model parameters. The specific models considered are the quasi-static cooling loop model developed by van den Oord \& Mewe (1989) and the two-ribbon flare model developed by Kopp \& Poletto (1984).

\subsection{Quasi-static Cooling Modeling}

In the quasi-static cooling loop model approximation (see van den Oord \& Mewe 1989 for a detailed discussion) one assumes that the flaring plasma decays through a sequence of stationary constant pressure loops (hence the name quasistatic cooling); in particular it assumed that the scaling laws that relate pressure, peak temperature, length and (volumetric) heating of such a loop, are also valid for the flaring plasma. For the special case of a cooling law of the form $\Lambda(T) \sim T^{1 / 4}$, which approximately applies at temperatures above $\log T \sim$ 7.2, van den Oord \& Mewe (1989) derive the following equation for the temperature evolution of the plasma:

$$
\frac{d T}{d t}=\frac{8}{21} T^{-13 / 8}\left(\frac{1}{\chi}-T^{7 / 2}\right)
$$

In Equation (1) the variable $t$ expresses time in terms of the radiative cooling time

$$
\tau=\frac{3 k T_{0}}{n_{0} \Lambda\left(T_{0}\right)}
$$

where $T_{0}$ denotes the temperature at the start of the decay phase, $n_{0}$ denotes the density, $\Lambda\left(T_{0}\right)$ denotes the cooling function, $k$ is Boltzmann's constant as usual, and $T$ is a dimensionless temperature expressing temperature relative to the plasma temperature $T_{0}$ at the beginning of the flare decay phase. Finally the parameter $1 / \chi=\left(T_{e} / T_{0}\right)^{7 / 2}$ is a dimensionless parameter allowing the presence of continued heating during the decay phase; for the case of no heating we have $\chi=\infty$ since then the final temperature $T_{e}$ of the flare plasma will tend to zero (see van den Oord \& Mewe 1989 for details). van den Oord \& Mewe (1989) also derive a solution for equation (1), which reduces for the case for no heating (i.e., $1 / \chi=0$ ) to the particularly simple form

$$
T=T_{0}(1+t / 3)^{-8 / 7} \text {. }
$$

For the total radiative output of the flare (which can be measured with an X-ray telescope) one finds-in this case-

$$
E_{\mathrm{rad}}=E_{0}(1+t / 3)^{-4} .
$$

Interpreting the flare decay light curve of EV Lac (see Fig. 3) in the context of the quasi-static cooling model one does not require the presence of any additional heating during the decay phase, similar to the results obtained by van den Oord \& Mewe (1989) in their analysis of a long duration flare on Algol. In Figure 5, I show the observed EV light curve together with the best-fit decay light curve calculated from equation ( 3 ); in Table 1 , I list the derived parameters, where I distinguish between directly measured parameters (such as emission measure and decay time scale) and inferred parameters ( such as density and length scale). As is clear from Table 1, the inferred flare densi-

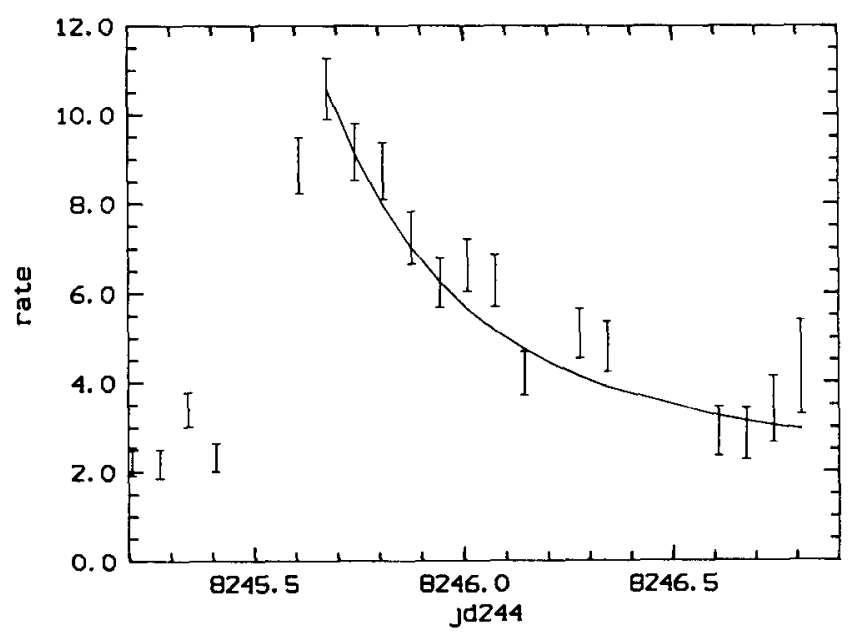

FIG. 5.-ROSAT all-sky survey light curve of the EV Lac long-duration flare together with the best-fit quasi-static cooling model as calculated from eq. (3); the model parameters are summarized in Table 1. 
TABLE 1

Quasistatic CoOling Physical Parameters

\begin{tabular}{|c|c|c|}
\hline Parameter & Value & Measured/Inferred \\
\hline Decay timescale (s) & 38380 & measured \\
\hline Temperature (MK) ...... & 30 & measured \\
\hline Emission measure $\left(\mathrm{cm}^{-3}\right)$ & $1.5 \times 10^{52}$ & measured \\
\hline Density $\left(\mathrm{cm}^{-3}\right) \ldots \ldots \ldots$ & $3 \times 10^{10}$ & inferred \\
\hline Pressure $\left(\right.$ dyn $\left.\mathrm{cm}^{-2}\right)$. & 194 & inferred \\
\hline Length $(\mathrm{cm}) \ldots \ldots \ldots$ & $6 \times 10^{11}$ & inferred \\
\hline Volume $\left(\mathrm{cm}^{3}\right)$ & $3 \times 10^{31}$ & inferred \\
\hline Thermal energy (ergs) & $9 \times 10^{33}$ & inferred \\
\hline
\end{tabular}

ties are relatively low, and therefore the inferred volume and length scales must be large. The reason for this is easy to understand: If the flare plasma cools predominantly through radiation (as is actually the case for the parameter range we are considering), the long decay timescale requires low densities (since radiative cooling is proportional to the square of the density). The length scales of the flaring plasma (as inferred from the scaling laws) are enormous; $L \sim 6 \times 10^{11} \mathrm{~cm}$ corresponds to $\sim 10$ stellar radii, and in fact exceeds the pressure scale height. Thus loops of that length cannot be constant pressure loops in contradiction to the original model assumption. To me this internal inconsistency does not appear too severe. First of all, scale height effects can be incorporated into the scaling law formalism (see Serio et al. 1981), and second, a significant fraction of the emission measure lost due to scale height effects could be recovered by considering expanding loop geometries. Before exploring such possibilities it is worthwhile, however, to pause and check whether the flare light curve can also be explained in the context of a different astrophysical scenario.

\subsection{Two-Ribbon Flare Modeling}

A totally different approach to the modeling for flare light curves has been taken by Kopp \& Poletto (1984) in their extension of reconnection theory to the modeling of two-ribbon flares. This model has been applied by Poletto, Pallavicini, \& Kopp (1989) to a long-duration flare observed on the flare star EQ Peg, and I follow their modeling strategy in the following. While one of the central assumptions of the van den Oord \& Mewe (1989) approach is that the flaring plasma maintains its physical identity, Kopp \& Poletto (1984) make exactly the opposite assumption and argue that during the flare decay continued heating is present, which leads to the appearance of freshly heated plasma in the flaring region. In contrast to the van den Oord \& Mewe (1989) model, Kopp \& Poletto (1984) identify the energy reservoir of the flare in terms of the energy difference between a nonpotential magnetic field and a potential magnetic field which is attained after reconnection; potential and nonpotential fields are separated by a neutral line which moves up during the flare progress. However, their model makes no provision for the conversion of magnetic energy into heating and radiative output; rather it has to be assumed that a certain fraction of the total released energy goes into soft X-rays. To be specific, the energy release rate is as- sumed to be of the form

$$
\begin{aligned}
& \frac{d E}{d t}=\frac{1}{8 \pi} 2 n(n+1)(2 n+1)^{2} R_{*}^{3} B_{m}^{2}\left[I_{12}(n) / P_{n}^{2}(\theta)\right] \\
& \times \frac{y_{1}^{2 n}\left(y_{1}^{2 n+1}-1\right)}{\left[n+(n+1) y_{1}^{2 n+1}\right]^{3}} \frac{d y_{1}}{d t} .
\end{aligned}
$$

In equation (4) $R_{*}$ and $B_{m}$ denote stellar radius and surface magnetic field respectively; the parameter $n$ denotes the order of the Legendre polynomial which is used to model the reconnected potential magnetic field, $I_{12}(n)$ and $P_{n}(\theta)$ relate to mathematical constants associated with these polynomials (see Kopp \& Poletto 1984 for details), and finally the function $y_{1}$ describes-in a dimensionless form - the upward motion of the neutral point through

$$
y_{1}=1+H_{m} / R_{*}\left[1-\exp \left(-t / t_{0}\right)\right],
$$

where $t_{0}$ is some characteristic time constant ( to be determined from the data), and $H_{m}$ denotes the maximum height reached by the reconnection point during the flare event; Poletto et al. (1989) assume $H_{m}$ to be given by the lobe width (which in turn is given by the zeros of the corresponding Legendre polynomial), and I follow their assumptions.

Poletto et al. (1989) show that the resulting flare decay light curves do not sensitively depend on the parameter $n$, i.e., the lobe width of the chosen magnetic field configuration. Specifically they show that the observed decay light curve of EQ Peg can be described by a wide variety of different magnetic field configurations, i.e., different values of $n$, and find that the decay phase of the EQ Peg light curve can be explained both by a large-scale magnetic configuration (i.e., small values of $n$ ) containing relatively low-density plasma as well as a compact configuration (i.e., large values of $n$ ) with high-density plasma. The rise phase, modeled following the description of Poletto et al. (1989) does, however, depend on the assumed lobe width $n$. In order to demonstrate this effect I have considered three hypothetical flares with the same value of decay time $t_{0}=90 \mathrm{ks}$ and lobes described by Legendre polynomials of order 2,6 , and 40 ; the light curves were then scaled to the same total radiative energy loss. The result of this calculation is shown in Figure 6,

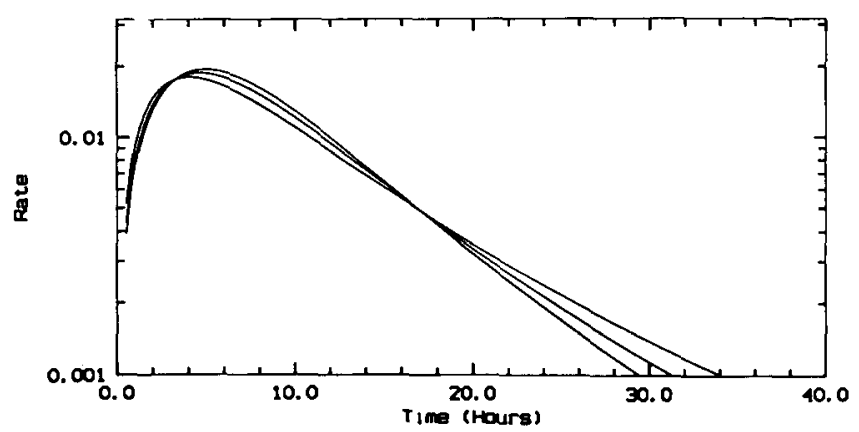

Fig. 6.-Temporal evolution of the flare emission from a two-ribbon flare as modeled from eqs. (4) and (5). For all curves the same decay $\left(t_{0}=\right.$ $90 \mathrm{ks}$ and the same total radiative flux was assumed. In Fig. 6 the resulting light curves for the case $n=40$ (top curve), $n=6$ (middle curve), and $n=2$ (bottom curve), respectively, are shown. 
which shows the time evolution of the flare emission for $n=40$ (top curve), $n=6$ (middle curve), and $n=2$ (bottom curve). As pointed out by Poletto et al. (1989), the decay phase light curves do indeed look very similar with almost the same slopes in a logarithmic representation. There are, however, pronounced differences in the time taken to reach flare maximum; the models with larger values of $n$ tend to peak earlier after flare onset and decay more slowly. Therefore, if flare onset and maximum are observed, a preferred choice between various values of $n$ can be made.

In Figure 7, I plot the observed light curve of EV Lac together with the best-fit two-ribbon flare models calculated from equations ( 4 ) and (5) for the cases $n=2,6$, and 40; in the fit process, the flare decay constant $t_{0}$, the time of flare onset and the overall normalization were used as free parameters. From a statistical point of view, all of the fits are acceptable with a slight preference for the case $n=2$. This case has the additional advantage that flare onset occurred at some time in between a data gap and not immediately after the last quiescent data point; again, if one had better information on the time of flare onset, a better determination of the lobe parameter $n$ could be made. The required surface magnetic field strengths range between 2000 and $5000 \mathrm{G}$, which is certainly consistent with magnetic field measurements on dMe stars (see Saar 1988); the major physical difference between the various models is the density, which ranges from $3 \times 10^{12} \mathrm{~cm}^{-3}$ for the case of $n=40$ to $5 \times 10^{10} \mathrm{~cm}^{-3}$ for $n=2$. These densities are quite uncertain and basically constitute order-ofmagnitude estimates by assuming a semicylindrical arcade with longitudinal extent of 1.5 the vertical extent (see Poletto et al. 1989).

\subsection{Comparison between the Modeling Results}

Figures 5 and 7 demonstrate that two models which significantly differ in their assumptions about the physical parameters of the flaring plasma can account for the observed X-ray light curve; in particular, we cannot distinguish between the

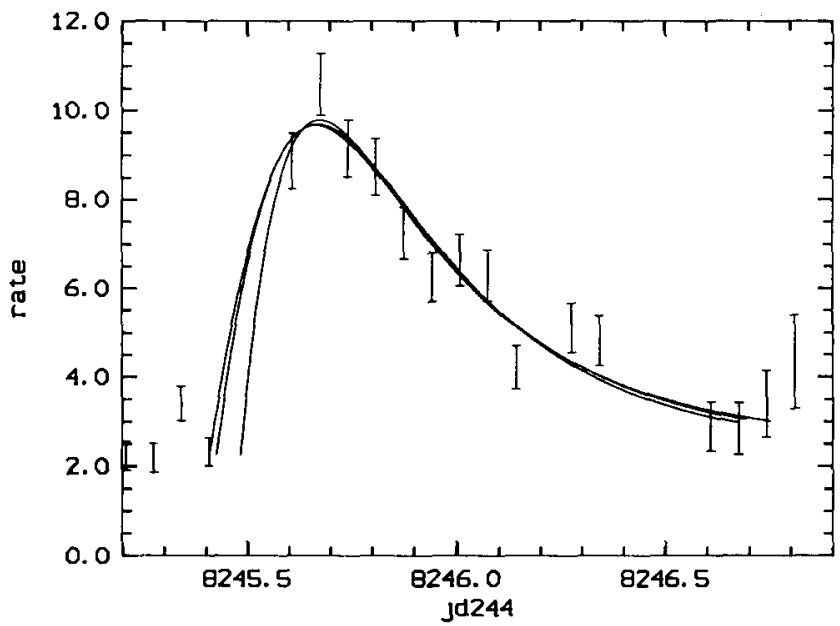

FIG. 7. - Best-fit two-ribbon flare light curves for the EV Lac long-duration flare calculated for the cases $n=40, n=6$, and $n=2$, respectively. Note that the curves look nearly indistinguishable during the flare decay, but differ substantially in the flare rise. case of a-by comparison-low-density plasma organised on rather large spatial scales from that of a compact high-density plasma. None of the models considered above appear to be fully satisfactory. The quasistatic cooling model leads to very large spatial scales in excess of a pressure scale height; a tworibbon flare model with large lobes leads to densities too low for internal consistency of the model ( since the released energy must be dissipated rapidly); and a two-ribbon flare with small lobe and high density forces flare onset shortly after the last pre-flare scan. Another potential problem with a compact flare interpretation is the absence of eclipses in the flare decay light curve. The rotation period of EV Lac is 4.378 days ( see Pettersen 1983 ) hence EV Lac rotates by $\sim 90$ degrees during the flare decay. If the flare is compact, the absence of eclipses implies that it must be located either in the circumpolar region or in that quarter of the stellar surface visible all the time. While this is clearly not a particularly stringent restriction for this flare on EV Lac, long-duration flares without eclipses have also been observed on other stars ( see Kürster \& Schmitt 1994); if such events are indeed compact, one should eventually detect eclipses through rotational modulation.

In the case of solar long duration flares one infers the presence of continuous heating by comparing the radiative cooling timescale (which requires simultaneous measurements of temperature, volume and emission measure) to the observed flare decay timescale ( see Moore et al. 1980). If such solar flares can be indeed used as a guide line for stellar long-duration flares, one would conclude that the models with continuous heating are to be preferred. Such models still differ enormously in the required densities, and a direct measurement of density would remove the ambiguities in our flare models. It is important to realize that the next generation of $\mathrm{X}$-ray telescopes will be able distinguish between the low- and high-density scenario for long duration stellar flares; the grating spectrometer to be flown on AXAF will have sufficient spectral resolution to measure density-sensitive line ratios in the required temperature range.

\section{WHAT IS THE SHORTEST TIMESCALE OF VARIABILITY?}

Considerable attention has been paid to the question of the shortest variability timescales of stellar flares. Extensive studies at optical wavelengths have been carried out by Russian astronomers using the $6 \mathrm{~m}$ telescope to obtain data with extremely high time resolution; these studies established $0.1 \mathrm{~s}$ as the shortest observed time scale of stellar flares (see Tovmasyan \& Zalinyan 1988). At X-ray wavelengths considerable attention has been devoted to the existence of microflares and the question whether the coronae of active stars are really "quiescent" or whether their quiescence can be interpreted simply as a succession of an overlapping multitude of flares ( $\mathrm{cf}$. Butler et al. 1986 and Pallavicini et al. 1990a for opposing views on this subject).

At X-ray wavelengths variability on short timescales is not readily detectable. Although most observations are carried out in a photon counting mode with individual counting events being time-tagged with an accuracy of $\sim$ milliseconds or better, the data, when binned into, say, $0.1 \mathrm{~s}$ time bins, are photon-limited. To be specific, let us consider the example of EV Lac (see Fig. 3), which in fact is the flare star on which optical micro-flashes with timescales of $0.1 \mathrm{~s}$ were detected, and ex- 
plore the observational requirements to detect hypothetical Xray flares on the same timescale. EV Lac's quiescent PSPC count rate is observed to be 2.2 counts $\mathrm{s}^{-1}$, i.e., 0.22 counts bin $^{-1}$ (assuming $0.1 \mathrm{~s}$ bins). If one demands that-with a confidence of $99.9 \%$-there is no spurious $0.1 \mathrm{~s}$ flare in an observation of, say, $10 \mathrm{ks}$ (i.e., $10^{5}$ bins), the number of photons $n$ required to detect such an event must satisfy $P(n, x)$ $=\left(\sum_{i=0}^{n} x^{i} e^{-x} / n !\right)^{100000}>1-10^{-3}$. As demonstrated in Table 2 , the function $P(n, x)$ depends sensitively on both $n$ and $x$. Determining $n$ for the case of $x=0.22$, one finds $n>6$, i.e., one requires instantaneous count rates in excess of 60 counts $\mathrm{s}^{-1}$. While such count rates are in principle detectable, the difficulty in practice lies in the fact, that first, the "background" level is usually not constant over such long time scales and spurious "flares" can be introduced by a time-variable background (see Press \& Schechter 1974), and second, one has no a priori knowledge of the relevant timescales of a given flare to be detected, and therefore many different binnings (i.e., time scales) of the data have to be considered.

Pallavicini, Stella, \& Tagliaferri (1990a) analyzed a long EXOSAT observation of UV Ceti with a power spectrum analysis and found that the measured power spectrum (see their Fig. 27) of UV Ceti could be interpreted as flickering (i.e., slope of power spectrum $\alpha \sim 1$ ), which becomes constant (i.e., turns into white noise) at frequencies above $2 \mathrm{mHz}$. This high-frequency turnover is caused by the photon statistics (the EXO$S A T$ count rate of UV Ceti was typically $\sim 0.05$ counts $\mathrm{s}^{-1}$ ), and implies that no excess power (over white noise) on timescales of $500 \mathrm{~s}$ or less is present in the EXOSAT data in a statistically significant fashion. One method to produce a flicker noise power spectrum is by superposition of individual "shots" with different duration and amplitudes; as pointed out by Pallavicini et al. (1990a), if microflares can indeed be identified by such "shots", no typical time scales and energies can be identified for such events.

A question left open from the $E X O S A T$ observation was whether a more sensitive observation would retrieve the flicker noise and allow to follow it to lower frequencies. The ROSAT XRT (which sees UV Ceti more than an order of magnitude brighter than $E X O S A T$ ) is an ideal instrument to do this; note, however, that $R O S A T$ observations cannot yield reliable power spectra at frequencies below $\sim 1 \mathrm{mHz}$, since a typical contiguous $R O S A T$ observation lasts about 1500 seconds. In Figure 8 , I show the mean power spectrum obtained from a 25 ks pointed ROSAT observation of UV Ceti together with a best-fit model; similar to Pallavicini et al. (1990a) the power spectrum has been fitted using a power law with a slope fixed at

TABLE 2

SAMPLE VAlues For $P(n, x)$

\begin{tabular}{llllll}
\hline$n / x$ & \multicolumn{1}{c}{0.1} & \multicolumn{1}{c}{0.2} & \multicolumn{1}{c}{0.3} & \multicolumn{1}{c}{0.4} & \multicolumn{1}{c}{0.5} \\
\hline $1 \ldots$ & 0.0 & 0.0 & 0.0 & 0.0 & 0.0 \\
$2 \ldots$ & $1.9 \times 10^{-7}$ & 0.0 & 0.0 & 0.0 & 0.0 \\
$3 \ldots$ & 0.68 & $3.4 \times 10^{-3}$ & $2.8 \times 10^{-12}$ & $1.9 \times 10^{-34}$ & 0.0 \\
$4 \ldots$ & 0.992 & 0.798 & 0.206 & $2.2 \times 10^{-3}$ & $3.3 \times 10^{-8}$ \\
$5 \ldots$ & 0.99987 & 0.9925 & 0.9246 & 0.667 & 0.24 \\
$6 \ldots$ & $>0.99999$ & 0.99979 & 0.99988 & 0.977 & 0.90 \\
$7 \ldots$ & $>0.99999$ & $>0.99999$ & $>0.99999$ & 0.9988 & 0.9937 \\
$8 \ldots$ & $>0.99999$ & $>0.99999$ & $>0.99999$ & 0.99995 & 0.99966 \\
\hline
\end{tabular}

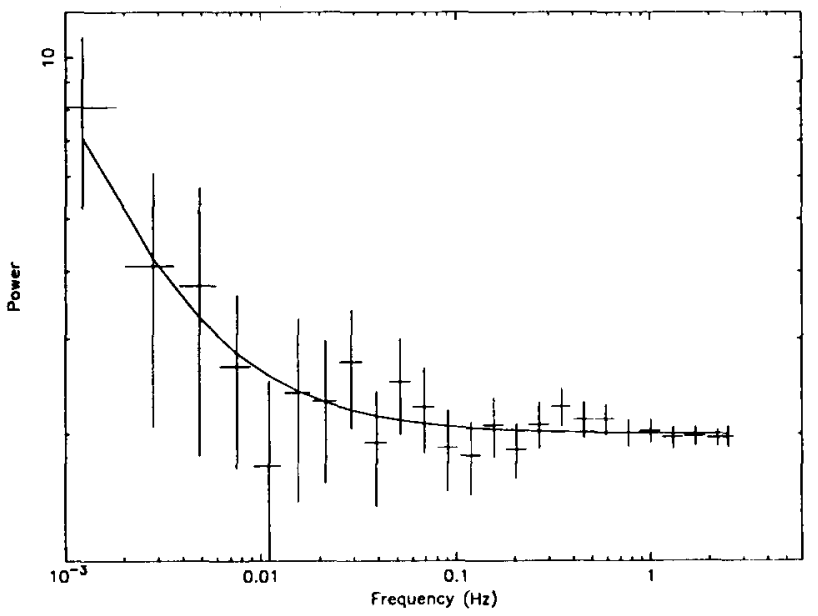

FIG. 8.-Power spectrum of a $25 \mathrm{ks}$ pointed ROSAT observation of the flare star UV Ceti in the frequency range $1 \mathrm{mHz}$ to $2 \mathrm{~Hz}$. Note the flicker noise component at lower frequency and the turnover to white noise.

the flicker value, and a constant ( fixed at the white noise value of 2), so that only one parameter, i.e., the normalization of the power law distribution, was adjusted. As is clear from Figure 8, we again recover the flicker component discovered by Pallavicini et al. (1990a), which we can however follow up to frequencies of $10 \mathrm{mHz}$. As a result of these new ROSAT observations we can state that no significant variability power is present at frequencies below $0.01 \mathrm{~Hz}$.

The last statement does of course not imply that in isolated events variability on shorter timescales may not be present. In particular, the problems caused by the photon-limited signal from typical stellar flare observations can be avoided if simultaneous observations with larger signal-to-noise ratio at other wavelengths (usually in the optical) are available; in this case the effective look window can be very much shortened and the question is reduced to the question of whether there are any deviations of the X-ray count from uniformity related to the optical flare.

Recently, Schmitt, Haisch, \& Barwig ( 1993a) presented an example of such an observation of a flare on the nearby flare star UV Ceti. During one of the observation intervals with simultaneous optical and X-ray coverage, two small flares were observed in the optical. One event was impulsive only with an overall timescale of $10 \mathrm{~s}$, the other event showed an impulsive phase followed by a gradual phase on a timescale of $\sim 5$ minutes. Surprisingly, the X-ray light curve showed small peaks approximately $30 \mathrm{~s}$ after the optical impulsive bursts; a plot of the simultaneously recorded X-ray and $B$-band light curve can be found in Schmitt et al. (1993a). Unfortunately, the small number of counts prevents any far-reaching physical conclusions; in fact, there is a small chance (estimated to be $\sim 10^{-3}$ by Schmitt et al. 1993a) that any one of the events detected on UV Cet is spurious. The more intriguing possibility is of course to assume that the events are real, in which case for the first time the impulsive phase of a stellar flare would have been detected at X-ray wavelengths. Since for solar flares the impulsive phase is the one relevant for particle acceleration, we may therefore have just begun to explore the conditions for particle acceleration in stellar flare environments. 
Ambruster, C., Snyder, W. A., \& Woods, K. S. 1984, ApJ, 284, 270

Barstow, M. A., Bromage, G. E., Pankiewicz, G. S., Gonzalez-Riestra, R., Denby, M., \& Pye, J. P. 1992, Nature, 353, 635

Bromage, G. E., et al. 1992, in ASP Conf. Ser. 26, 7th Cambridge Workshop on Cool Stars, Stellar Systems, and the Sun, ed. M. S. Giampapa \& J. A. Bookbinder (San Francisco: ASP), 80

Butler, J. L., Rodonò, M., Foing, B. H., \& Haisch, B. M. 1986, Nature, 321,679

deJager, C., et al. 1986, A\&A, 156, 95

Doyle, J., et al. 1991, MNRAS, 248, 503

Fleming, T. A., Giampapa, M. S., Schmitt, J. H. M. M., \& Bookbinder, J. A. 1993, ApJ, 410, 387

Haisch, B. M. 1983, in Activity in Red Dwarf Stars, ed. P. B. Byrne \& M. Rodonò (Astrophysics and Space Science Library, 102), 255

Haisch, B. M., Linsky, J. L., Bornmann, P. L., Antiochos, S. K., Golub, L., \& Vaiana, G. S. 1983, ApJ, 267, 280

Haisch, B. M., Strong, K. T., \& Rodonò, M. 1991, ARA\&A, 29, 275

Kopp, R. A., \& Poletto, G. 1984, Sol. Phys., 93, 351

Kürster, M., \& Schmitt, J. H. M. M. 1994, in preparation

Linsky, J. L. 1985, Sol. Phys., 100, 333

Moore, R., et al. 1980, in Solar Flares, ed. P. A. Sturrock (Boulder: Colorado Associated Univ. Press)

Pallavicini, R., Stella, L., \& Tagliaferri, G. 1990a, A\&A, 228, 443
Pallavicini, R., Tagliaferri, G., Pollock, A. M. T., Schmitt, J. H. M. M., \& Rosso, C. 1990b, A\&A, 227, 483

Pettersen, B. 1983, in Activity in Red Dwarf Stars, ed. P. B. Byrne \& M. Rodonò (Astrophysics and Space Science Library, 102), 17

Poletto, G., Pallavicini, R., \& Kopp, R. A. 1989, A\&A, 201, 93

Press, W. H., \& Schechter, P. 1974, ApJ, 193, 437

Rieger, E. 1993, private communication

Saar, S. H. 1988, ApJ, 324, 141

Schmitt, J. H. M. M., Collura, A., Sciortino, S., Vaiana, G. S., Harnden, F. R., Jr., \& Rosner, R. 1990, ApJ, 365, 307

Schmitt, J. H. M. M., \& Rosso, C. 1988, A\&A, 191, 99

Schmitt, J. H. M. M., Haisch, B. M., \& Barwig, H. 1993a, ApJ, in press Schmitt, J. H. M. M., Zinnecker, H., Cruddace, R., \& Harnden, F. R., Jr. 1993b, ApJ, 402, L13

Serio, S., Peres, G., Vaiana, G. S., Golub, L., \& Rosner, R. 1981, ApJ, 243, 288

Stern, R. A., et al. 1992, ApJ, 391, 760

Tovmasyan, G. M., \& Zalinyan, V. P. 1988, Astrofizika, 28, 131

Tsuru, T., et al. 1989, PASJ, 41, 679

van den Oord, G. H. J., \& Mewe, R. 1989, A\&A, 213, 245

van den Oord, G. H. J., Mewe, R., \& Brinkman, A. C. 1989, A\&A, 205, 181 